\title{
DEVELOPMENT OF SUPERCONDUCTING CONDUCTORS FOR LARGE HELICAL DEVICE
}

\section{T. Mito, J. Yamamoto, K. Takahata, N. Yanagi, O. Motojima, and LHD Design Group}

National Institute for Fusion Science (NIFS)

Furo-cho, Chikusa-ku, Nagoya-shi, 464-01, Japan

\section{Abstract}

Superconducting helical coils of the Large Helical Device (LHD) requires the superconducting conductors with large current capacity from $20 \mathrm{kA}$ to $30 \mathrm{kA}$ and high current density of $55 \mathrm{~A} / \mathrm{mm}^{2}$ at $8 \mathrm{~T}$. NbTi superconductor with pool boiling is selected considering the large electromagnetic force and the complicated helical windings. Several conductors are designed aiming to study how the difference of the position of pure aluminum in the conductors affects the stability and the mechanical properties. Scale-down R\&D conductors with the operational current from $7 \mathrm{kA}$ to $10 \mathrm{kA}$ were made on an experimental basis. We have been testing these scale-down conductors about the superconducting characteristics, the stability and the mechanical properties. The detail of design and test results about the superconducting characteristics are described.

\section{Introduction}

The most urgent R\&D item, which is necessary for the L.HD design and fabrication, is to develop the most. adequate superconducting conductor for the LHD helical windings. $\mathrm{NbTi} / \mathrm{Cu}$ is selected for the superconducting material because of its good mechanical properties. The cooling type of the LHD helical coils has been decided to the pool boiling considering the advantage of the flexibility for complicated helical windings and the easy for conductors joints in the windings.

The LHD is a fully superconducting heliotron/torsatron type fusion experimental device. All coils of LHD (two helical coils and three pairs of poloidal coils) are designed to be superconducting. The present design parameters of LHD are; the major radius $3.9 \mathrm{~m}$, the minor radius of $0.975 \mathrm{~m}$, the helical coil number of 2 , the toroidal pitch number of 10 , the toroidal magnetic field of $3 \mathrm{~T}$ (in the first phase) and $4 \mathrm{~T}$ (in the second phase), and the stored magnetic energy of $0.9 \mathrm{GJ}$ (Phase I) and 1.6GJ (Phase II).

\section{Conductor Design}

\section{Basic Requirements}

The conductor for the helical coils must satisfy the conflicting requirements of the mechanical toughness after the winding and the flexibility during the winding. Because of the twisted helical windings, the coils are loaded by the large electromagnetic force of about $10 \mathrm{MN} / \mathrm{m}$ $(\mathrm{B}=4 \mathrm{~T}$ in Phase II ) in not only hoop direction but also transverse direction of the winding locus. It means that the conductor in the helical windings must withstand the large stress in all directions. The mechanical rigidity of the windings is also required in order to avoid magnetic surface destruction of LHD. On the contrary, the flexibility of the conductor is necessary to wind the helical coils keeping a required severe accuracy. The helical coils will be wound at the LHD experimental building because of the difficulty in their transportation from factories to the institute, which is another reason for the necessity of flexible conductors to reduce the construction time and efforts of the LHD helical coils.

The high current density of the helical coil package and the enough stability and safety of the coils are another conflicting requirements. The current density of the helical coils is an important parameter to improve the physics properties of LHD, especially to increase the boundary shear and to supply the enough room for divertor. The high current density of the conductor is required form the above reason. On the contrary, the conductor must be enough stable against the coil quench and safety after the quench because the coil system of LHD is one of the largest superconducting coil system in the world. The necessary area of the stabilizer restricts the current density of the conductor.

In Phase II experiment, the poloidal coils are operated in pulse mode. Pulse losses due to the field change of the poloidal coils $(0.04 \mathrm{~T} / \mathrm{s}$ at the position of helical coils) must be reduced in order not to affect the stability of the helical coils

\section{Designed Conductors for LHD Helical Coils}

There are two types of designed conductors for the LHD helical coils. One is proposed from the LHD coil design and the farther R\&D coil design in FY1989 by coil manufacturers (Design-Hr2, Design-M). The other is developed in the R\&D program on superconducting conductor for LHD in collaboration with superconducting cable manufacturers and researchers of universities (KISO-1B, 2B, 3B, 4B).

Figure 1 shows the cross sections of the designed conductors. These conductors are designed aiming to study how the difference of the position of pure aluminum in the conductors affects the stability and the mechanical properties. Design-Hr2 has a pure aluminum stabilizer at the side of the superconducting cable. Design-M has four copper clad aluminum stabilizers surrounding the superconducting cable. KISO-1B and KISO-4B have an aluminum stabilizer inside of the superconducting cable. $\mathrm{KISO}-2 \mathrm{~B}$ and $\mathrm{KISO}-3 \mathrm{~B}$ have distributed aluminum stabilizers for each strand using the newly developed aluminum-stabilized strands cable.

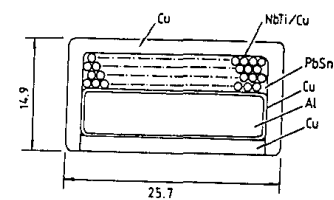

DESIGN-Hr2

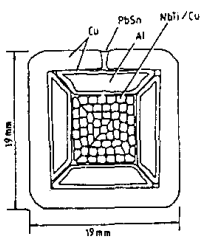

DES IGN-H

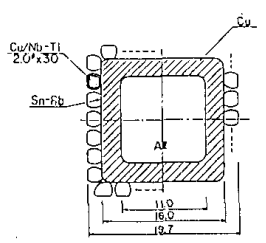

KISO-1B

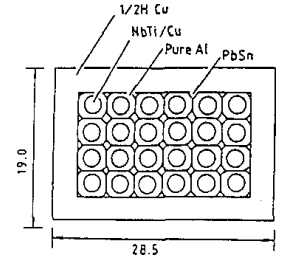

$K I S O-2 B$

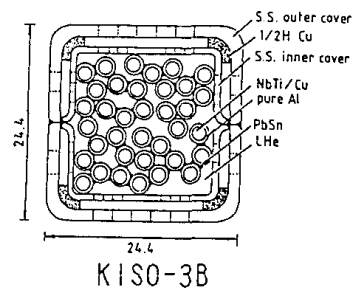

$\mathrm{K} 1 \mathrm{SO}-3 \mathrm{~B}$

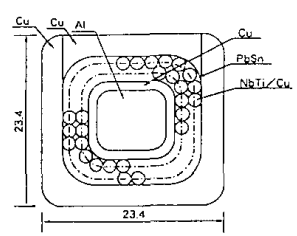

KISO- $4 \mathrm{~B}$
Figure 1. Cross sections of designed conductors for LHD

0018-9464/91/0300-2224\$01.00 @ 1991 IEEE 
Design-Hr2 is designed according to an experienced design pattern for a large current conductor; the compacted strands cable and the copper clad pure aluminum stabilizer are arranged parallel and are covered with half hard copper housing, these components are soldered together. The conductor has two layered strands cable in order to modify the aspect ratio of the conductor. Design- $M$ has been designed and fabricated for one of the R\&D coils, TOKI-MC, considering about the mechanical toughness, the stability, and manufacturing feasibility.

The positional relation of the superconducting cable and the aluminum of KISO-1B is the same as that of KISO-4B. The difference of both conductors is as follows. KISO- $1 \mathrm{~B}$ is made much account of the cooling stability. The superconducting strands are soldered on the copper clad aluminum and are directly cooled by liquid helium. On the other hand KISO-4B is set importance on the mechanical properties. The conductor is covered with a half hard copper sheath whose inside are round-cornered for its reinforcement.

KISO-2B consists of a newly developed

aluminum-stabilized compacted strands cable and a half hard copper sheath as a mechanical reinforcement. This conductor is designed so as to minimize the distance from the superconductor to the stabilizer. KISO-3B is using the same aluminum-stabilized wires with triplex cabling. This cable is covered with double stainless-steel conduits which have many holes for the liquid helium penetration. This conductor is cooled internally by the liquid helium penetrated into the conductor.

A monolithic superconducting wire can not be applied as a superconducting cable for a large current conductor from a viewpoint of the feasibility of manufacturing a long wire. Therefore the superconducting cable twisted with multiple strands becomes necessary. Twisting methods $0_{-}^{\circ}$ strands are classified into three patterns; 1) single layer strands cable, 2) multi-layered strands cable without transposition, and 3) triplex cable with transposition Since the resistivity of the each strand is zero, it is necessary to study how the twisting method affects the uniformity of the current distribution between strand. It can be feared that a lack of uniformity of the current distribution between strands may cause the degradation of the critical current of the conductor which is calculated as a simple sum of a strand critical current. KISO-1B has a single layer strands cable. DESIGN-Hr2 and DESIGN-M have multi-layered strands cables twisted same direction. KISO-2B and KISO-4B have multi-layered strands cables twisted opposite direction for each layer. KISO-3B has a triplex cable in which strands are transposed. So we can examine the difference of each twisting method.

\section{Development of Scale-down R\&D Conductors}

Scale-down R\&D conductor with the operational current from $7 \mathrm{kA}$ to $10 \mathrm{kA}$ have been made on an experimental basis. We have been testing these scale-down conductors about the superconducting characteristics, the stability and the mechanical properties. In this paper we describe the present situation of these tests which includes the measurement of the critical current and the stability.

\section{Structure of Scale-down R\&D Conductors}

The cross sections of the scale down conductors are similar figures of the designed conductor. Main parameters of the scale-down conductors are listed in Tab. 1 and those cross sections are shown in Fig. 2.

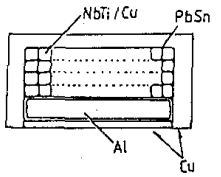

DESIGN-Hr2

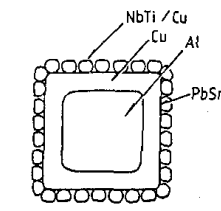

$\mathrm{K} / \mathrm{SO}-1 \mathrm{~B}$

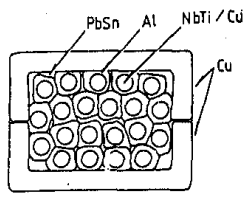

$K 1 S 0-2 B$

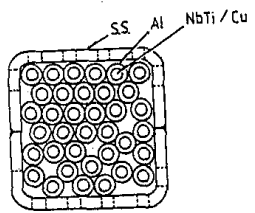

$\mathrm{K} / \mathrm{SO}-3 \mathrm{~B}$

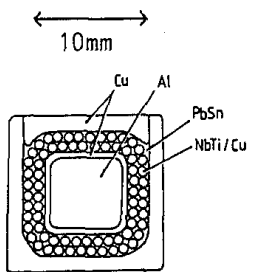

$\mathrm{K} \mid \mathrm{SO}-4 \mathrm{~B}$

Figure 2. Cross sections of scale down R\&D conductors

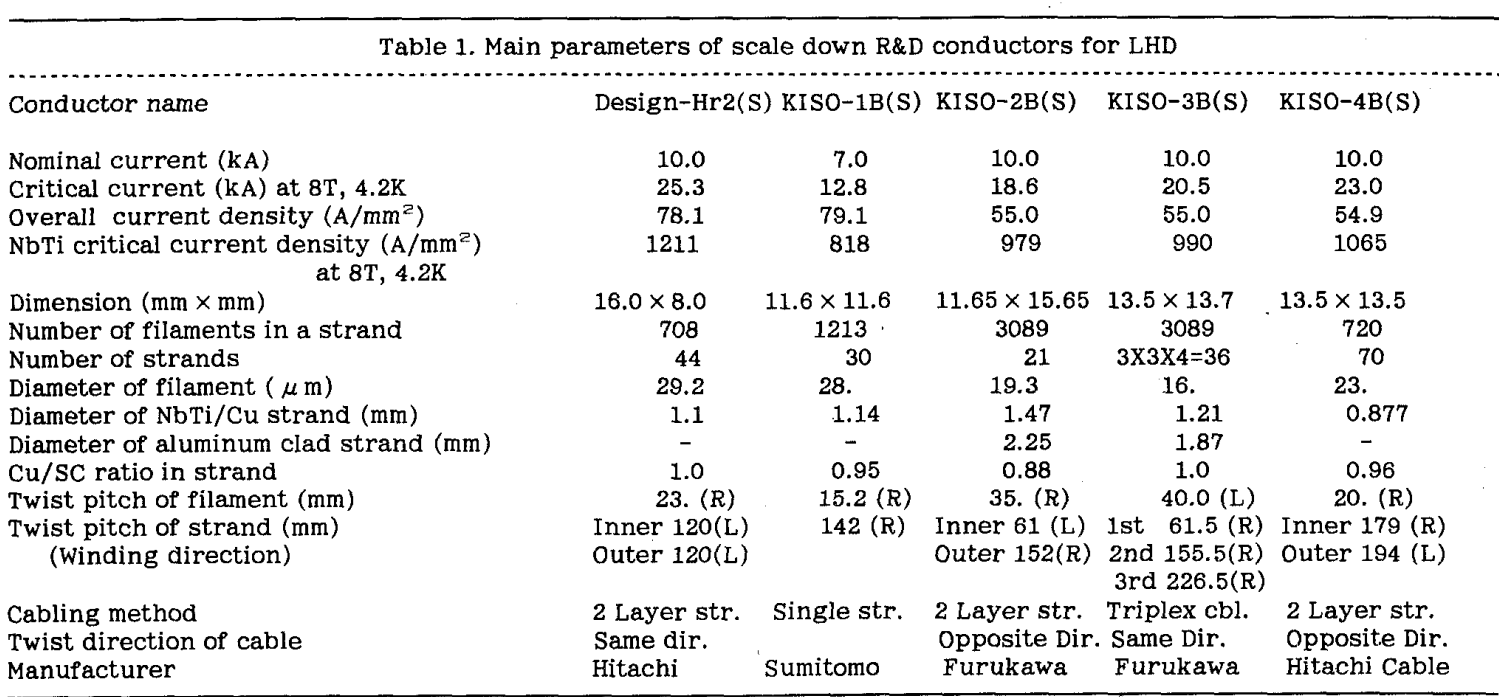


In Tab. 1 and Fig. 2, '(S)' after the conductor name means the scale down R\&D conductor. The composition of pure aluminum of Design-Hr2(S) is not similar reduction of Design-Hr2 in order to utilize the copper housing of the conductor of TOKI-HB (one of the R\&D coils presented in this conference). The stainless-steel conduit of $\mathrm{KISO}-3 \mathrm{~B}(\mathrm{~S})$ is simplified from double conduits to a single conduit for the convenience of manufacturing. Other minor changes of the cross section and the composition of the scale down conductors were done in order to utilize the existing materials.

\section{Experimental Method}

The superconducting characteristics are measured for the scale down conductors using the conductor test facility. The conductor test facility consists of the superconducting split coils for bias filed (the inner diameter: $120 \mathrm{~mm}$, the outer diameter: $334 \mathrm{~mm}$, the length of one coil: $100 \mathrm{~mm}$, the gap space: $30 \mathrm{~mm}$, the central field: $8 \mathrm{~T}$ ), the cryostat, the $30 \mathrm{kA}$ current leads, and the $30 \mathrm{kA}$ power supply. The setup inside the cryostat is shown in Fig. 3. Two short sample conductors are inserted into the split coils and are soldered at the bottom to make the going and returning sample current.

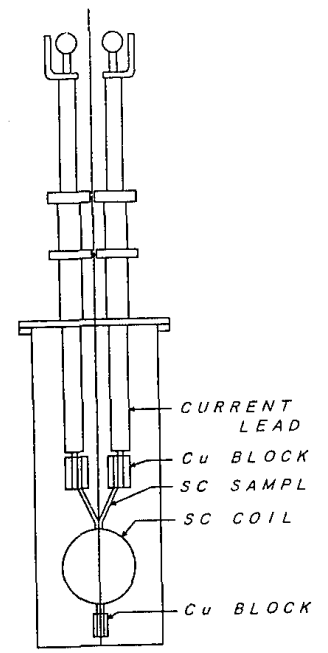

Figure 3. Setup of the conductor test facility

Many voltage taps, $\mathrm{Au}-\mathrm{Fe}$ thermocouples, and heaters were attached to the sample conductor as shown in Fig. 4. We can measure the voltage distribution and the normal zone propagation after the quench using the longitudinal voltage taps. The transverse voltage taps (E4a,b,c,d, etc.) were also attached to the sample conductor in order to measure the voltage distribution in the cross section of the conductor. The thermocouples are used for the measurement of temperature rise after the quench. The heater is necessary to initiate the quench for the measurement of the conductor stability.

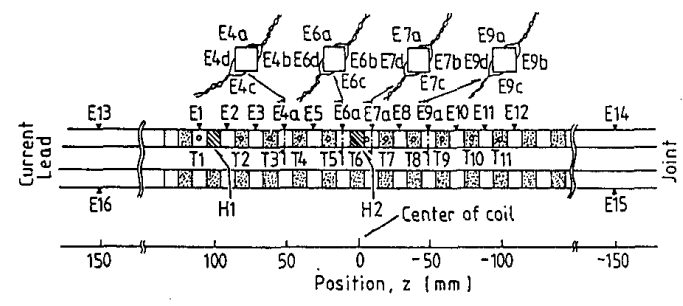

Figure 4. Measuring points of sample conductor

\section{Measurement of Critical Current}

The critical currents of each conductor have been measured to investigate how the difference of twisting method affects the critical current of the conductor. We can estimate the uniformity of the current distribution between strands by measuring the critical current.

The critical current of the conductor is reduced by the conductor self field. In the case of the conductors having a multi-layered superconducting cable, the self field and the critical current of each strand are different for each layer.

Figure 5 shows the measured quench current of Design-Hr2 versus the bias magnetic field. Where the dotted line shows the critical current of the conductor without the self field (the critical current of single strand times number of strands), the solid line is the critical current considering self field at each position of strand, the chain line is the critical current supposing that all strands have the same critical current which is defined by the maximum self field, and the circle marks show the measured quench current. The measured quench currents agree with the solid line. It means that the different current can flow for each strand up to the critical current corresponding to the strand position.

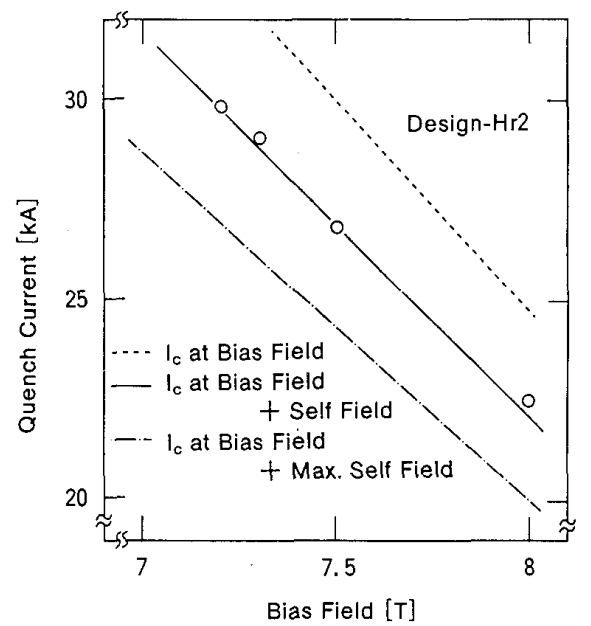

Figure 5. Quench current of Design-Hr2 vs. bias field

The same results about the critical current were observed for KISO-2B and $\mathrm{KISO}-4 \mathrm{~B}$ as shown in Fig. 6. The degradation of the quench current was observed for KISO-2B. This degradation was due to the mechanical disturbance. The sample conductors were excited to create the magnetic field in the opposite direction of the bias field so that the two sample conductors pushed one another. Therefore the sample does not need rigid supports. The mechanical disturbance, however, was induced by the insufficient support at the bottom joint of tre conductors.

The critical current of KISO-1B and KISO-3B could not be measured correctly. The current distribution of KISO-1B was very unbalanced because of the defective soldering in the conductor. So the conductor has quenched at a low current. KISO-3B was mechanically damaged at the first excitation test, and could not be excited up to the critical current.

The ramp rate dependence of the quench current is shown in Fig. 7. No degradation of the quench current is observed up to the rapid ramp rate of $400 A / s$. 


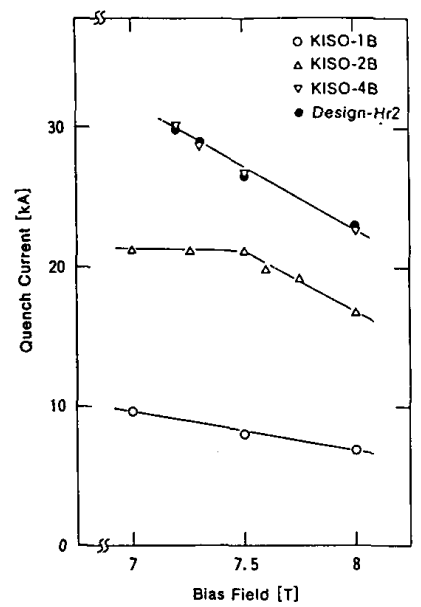

Figure 6. Quench current of the conductors vs. bias field

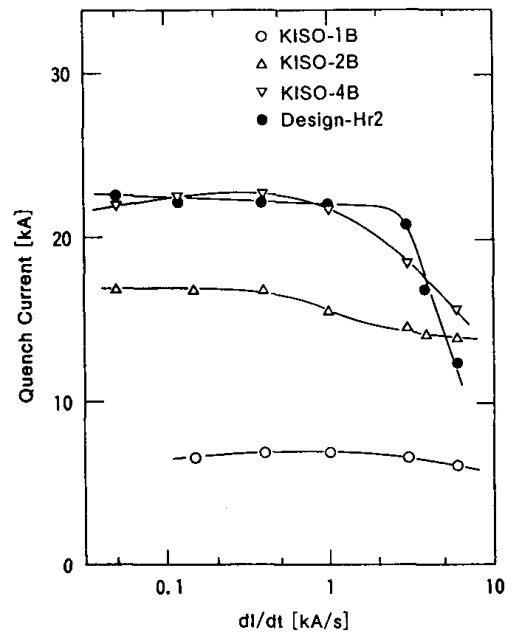

Figure 7. Quench current of the conductors vs. ramp rate

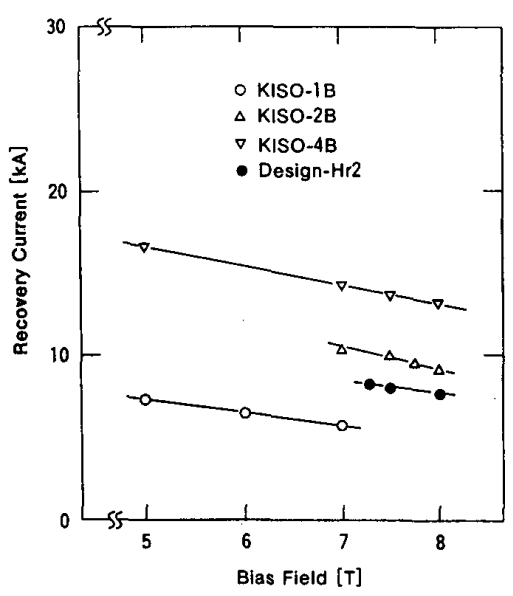

Figure 8. Recovery current of the conductors

\section{Measurement of Stability}

The quench recovery current of the conductors are shown in Fig. 8. The measured recovery currents agree with the calculated values using the normal resistivity measured by voltage signal after the quench as listed in Tab. 2. The normal resistivity of each conductor, however, is greater than the design value which is calculated from the resistivity of each element of the conductor. This reason is considered as the deterioration of aluminum conductivity and the insufficient soldering, because these sample conductors were fabricated on trial in a very short time.

Table 2. Recovery Current of the conductors at $8 \mathrm{~T}$

Conductor Name Design-Hr2 KISO-2B KISO-4B

$\begin{array}{llll}\text { Resistance (M) } \Omega / \mathrm{m} & 2.1 \times 10^{-6} & 2.3 \times 10^{-6} & 1.2 \times 10^{-6} \\ \text { Resistance (D) } \Omega / \mathrm{m} & 1.5 \times 10^{-6} & 1.0 \times 10^{-6} & 7.4 \times 10^{-7} \\ \text { Recovery (M) } \mathrm{kA} & 7.6 & 9.1 & 13.0 \\ \text { Recovery (C) } \mathrm{kA} & 7.9 & 9.3 & 13.0\end{array}$

Where '(M)' means measured value, '(D)' is design value, and '(C)' is calculated value using a measured resistance

\section{Discussion}

From the measurement of the critical current, we can say as follows.

1) The multi-layered strands cable without transposition is usable as a superconducting cable for a large current conductor.

2) Any degradation of the quench current caused by the unbalanced current distribution between the strands was not observed for the conductor with multi-layered strands cable.

3) The quench current of the conductor agrees with the critical current considering the effect of self field at the position of each strand.

4) No degradation of the quench current is observed up to the rapid ramp rate of $400 \mathrm{~A} / \mathrm{s}$

From the measurement of the stability, we can say as follows.

1) The design technique based on the existing stability criteria is applicable for a large current conductor.

2) No meaningful difference was observed in the stability according to the difference of the position of pure aluminum and superconducting cable.

\section{Conclusion}

We have been designed various superconducting conductors as a candidate for the LHD helical coils. Scale down R\&D conductors were manufactured and were tested about their critical current and stability. It is confirmed that a multi-layered strands cable is applicable for the superconducting cable and the position of pure aluminum does not affect the stability. We are planing to study the mechanical properties of the conductor, which are very important items for LHD design. After the investigation of the mechanical properties and the short sample test of the conductur in its actual size, the cross sectional structure of the conductor will be decided.

\section{Acknowledgements}

The authors are indebted to Prof. A. Iiyoshi, Director General of NIFS, for his continuous encouragements. The authors are also grateful to Dr. F. Sumiyoshi and Mr. N. Ohito of Kagoshima University, Dr. Tsukamoto and Dr. N. Amemiya of Yokohama National University, and Dr. T. Kawashima of Fukuoka Institute of Technology for their collaboration of design and test of conductors. 